The Astrophysical Journal, 686:1420-1425, 2008 October 20

(C) 2008. The American Astronomical Society. All rights reserved. Printed in U.S.A.

\title{
A LOW-ORDER SOLAR DYNAMO MODEL: INFERRED MERIDIONAL CIRCULATION VARIATIONS SINCE 1750
}

\author{
DÁrio Passos ${ }^{1}$ AND ILídio LoPEs ${ }^{1,2}$ \\ Received 2008 March 24; accepted 2008 July 2
}

\begin{abstract}
In this work we present the possible variations that the meridional circulation of the Sun might have undergone during the last 250 years. In order to do this, we reduce an $\alpha-\Omega$ dynamo to a low-order system that focuses on the time evolution of one of the solar magnetic field components. Afterward we used a method based on the analysis of phase space of the superficial toroidal magnetic field to infer changes in the superficial meridional circulation. We used sunspot numbers to build a time series that approximately represents the magnetic field behavior. After reconstructing the time series' phase space we assume equilibrium solutions for each solar cycle and we fit them to our model. The resulting fit parameters are shown to depend on background quantities of the theoretical model, such as magnetic diffusivity, differential rotation, meridional circulation, etc. The methodology presented here allows one to extract information about the meridional circulation average behavior, and possibly other parameters, from more that 250 years of sunspot number observations.
\end{abstract}

Subject headings: Sun: general — Sun: magnetic fields — sunspots

\section{INTRODUCTION}

Although the average behavior of the solar cycle can be explained by current dynamo theories, the variation in amplitude and period of the 11 year sunspot cycle is still hard to understand (Charbonneau 2005). This matter assumes an important role if we have in mind that the solar cycle variability has a massive influence on the interplanetary space that surrounds Earth and possibly an impact on the Earth's climate (Haigh 1996; Marsh $\&$ Svensmark 2003). Presently, where climate change is a very debated subject, telecommunication satellites are indispensable, and the advent of an active human solar system exploration takes shape, solar variability assumes a special role in this broad picture. Present solar variability studies indicate that the complicated structure and interaction of magnetic fields in the convection zone and lower solar atmosphere might be the roots of several solar phenomena that occur around the solar cycle peak (Livingston 1982; Harvey \& White 1999). Dynamo theories try to explain the overall behavior of the solar magnetic fields, but the complex nature of the equations makes it difficult to account for all possible effects.

One of the most studied types of dynamo is the BabcockLeighton dynamo model that assumes that the $\alpha$-effect (the recycling mechanism of toroidal to poloidal magnetic field) is due to the decay of bipolar sunspot pairs (Babcock 1961). An evolution of these dynamo models are the so-called flux-transport dynamos (Ossendrijver 2003) which include a plasma flow that, at the surface, transports small magnetic features from the equator to the poles, the so-called meridional circulation. Theoretically, this flow acts as a clock regulating some of the features observed during the solar cycle (e.g., its period and possibly amplitude; Dikpati \& Charnonneau 1999; Nandy \& Choudhuri 2002; Chatterjee et al. 2004; Charbonneau 2005; Dikpati et al. 2006). Experimental measurements of this plasma flow using helioseis-

\footnotetext{
${ }^{1}$ CENTRA, Departmento de Física, Instituto Superior Técnico, Avenida Rovisco Pais, 1049-001 Lisboa, Portugal; dariopassos@fisica.ist.utl.pt; lopes@ fisica.ist.utl.pt.

2 Departamento de Física, Universidade de Évora, Colégio António Luis Verney, 7002-554 Évora, Portugal.
}

mic techniques estimated values for its velocity around $20 \mathrm{~m} \mathrm{~s}^{-1}$ at the surface (poleward directed) and $3 \mathrm{~m} \mathrm{~s}^{-1}$ (equatorward directed) at the base of the convective zone (Giles et al. 1997; Hathaway et al. 2003).

Priest (1984) showed that some of the dynamics of the solar dynamo can be explained in terms of dynamical systems analysis. Features, like magnetic field reversal, have also been recently observed in laboratory dynamo experiments (Berhanu et al. 2007; Dubrulle et al. 2007) which indicate that, within certain approximations, some of these dynamics can be explained by low-order dynamo models (Mininni et al. 2001; Pontieri et al. 2003; WilmotSmith 2005).

In this work we use a low-order dynamo model associated with sunspot counts to infer properties of the meridional circulation over time. First, we reduce the equation set of a generic axisymmetric $\alpha-\Omega$ dynamo to a low-order dynamo model. We focus our attention on the temporal behavior of the toroidal component of the magnetic field at the surface of the Sun.

Second, we reconstruct the phase space of a time series that corresponds to this toroidal component, using observations of the sunspot number for the last 250 years. We assume that the behavior over time of the sunspot number is the same as the toroidal field component which is considered to be its source. Moreover, we fit our model to the phase space of the calculated time series by assuming equilibrium solutions for each solar cycle. This allows us to infer variation in the meridional circulation at the surface for this 250 year period. Finally, we present our results, discuss them in terms of present dynamo research, and speculate a little about possible correlations between some sharp variation episodes and other relevant observational phenomena.

\section{LOW-DIMENSIONAL DYNAMO MODEL}

\subsection{Oscillatory Behavior of the Toroidal Magnetic Field Component}

The mean solar magnetic field $\overline{\boldsymbol{B}}$ can be expressed in terms of its toroidal and poloidal components, $\overline{\boldsymbol{B}}=\boldsymbol{B}_{\phi}^{\prime}+\boldsymbol{B}_{p}^{\prime}$, where $\boldsymbol{B}_{p}^{\prime}=$ 
$\nabla \times\left(A_{p}^{\prime} \hat{\boldsymbol{e}}_{\phi}\right)$. Using the induction equation and spherical symmetry, we get the following equations set for an axisymmetric dynamo model (Charbonneau 2005),

$$
\begin{aligned}
\frac{\partial B_{\phi}^{\prime}}{\partial t}= & \eta\left(\nabla^{2}-\frac{1}{\bar{r}^{2}}\right) B_{\phi}^{\prime}+\frac{1}{\bar{r}} \frac{\partial\left(\bar{r} B_{\phi}^{\prime}\right)}{\partial r} \frac{\partial \eta}{\partial r}-\bar{r} \boldsymbol{v}_{p} \cdot \nabla\left(\frac{B_{\phi}^{\prime}}{\bar{r}}\right) \\
& -B_{\phi}^{\prime} \nabla \cdot \boldsymbol{v}_{p}+\bar{r}\left[\nabla \times\left(A_{p}^{\prime} \hat{\boldsymbol{e}}_{\phi}\right)\right] \cdot \nabla \Omega, \\
\frac{\partial A_{p}^{\prime}}{\partial t}= & \eta\left(\nabla^{2}-\frac{1}{\bar{r}}\right) A_{p}^{\prime}-\frac{\boldsymbol{v}_{p}}{\bar{r}} \cdot \nabla\left(\bar{r} A_{p}^{\prime}\right),
\end{aligned}
$$

where $\bar{r}=r \sin \theta, \nabla \Omega$ represents the differential rotation of the Sun, $\boldsymbol{v}_{p}$ is the meridional circulation, and $\eta$ is the magnetic diffusion. For simplification we assume that $\eta$ is a constant over all the convection zone, so that $\partial \eta / \partial r=0$ and the plasma is incompressible. We then get

$$
\begin{aligned}
\frac{\partial B_{\phi}^{\prime}}{\partial t}= & -\bar{r} \boldsymbol{v}_{p} \cdot \nabla\left(\frac{B_{\phi}^{\prime}}{\bar{r}}\right)+\bar{r}\left[\nabla \times\left(A_{p}^{\prime} \hat{\boldsymbol{e}}_{\phi}\right)\right] \cdot \nabla \Omega \\
& +\eta\left(\nabla^{2}-\frac{1}{\bar{r}^{2}}\right) B_{\phi}^{\prime}-\Gamma\left(B_{\phi}^{\prime}\right) B_{\phi}^{\prime}, \\
\frac{\partial A_{p}^{\prime}}{\partial t}= & -\frac{1}{\bar{r}} \boldsymbol{v}_{p} \cdot \nabla\left(\bar{r} A_{p}^{\prime}\right)+\alpha B_{\phi}^{\prime}+\eta\left(\nabla^{2}-\frac{1}{\bar{r}^{2}}\right) A_{p}^{\prime},
\end{aligned}
$$

where we also introduced a generic $\alpha$-effect to explain the conversion of toroidal to poloidal field and a term that accounts for the removal of magnetic flux from the bottom of the convection zone by magnetic buoyancy. Following the suggestions of Pontieri et al. (2003), we assume that the removal rate is proportional to the magnetic buoyancy, $\Gamma \sim \gamma B \prime_{\phi}^{2} / 8 \pi \rho$, where $\gamma$ is a constant and $\rho$ is the plasma density.

We can now separate the temporal from the spatial behavior of the fields by performing a variable separation in the following way,

$$
\begin{aligned}
B_{\phi}^{\prime}(r, \theta, t) & =b_{\phi}(r, \theta) B_{\phi}(t)=b_{\phi} B_{\phi}, \\
A_{p}^{\prime}(r, \theta, t) & =a_{p}(r, \theta) A_{p}(t)=a_{\phi} B_{p} .
\end{aligned}
$$

Using equations (3) and (4) in equations (1) and (2) we get

$$
\begin{gathered}
\frac{d B_{\phi}}{d t}=c_{1 b} B_{\phi}+c_{2 a} A_{p}-c_{3 b} B_{\phi}^{3}, \\
\frac{d A_{p}}{d t}=c_{1 a} A_{p}+\alpha c_{2 b} B_{\phi},
\end{gathered}
$$

where we have the spatial coefficients, $c_{n m}(r, \theta)$, defined as

$$
\begin{gathered}
c_{1 a}=\eta\left(\frac{1}{a_{p}} \nabla^{2} a_{p}-\frac{1}{\bar{r}^{2}}\right)-\frac{1}{\bar{r} a_{p}} \boldsymbol{v}_{p} \cdot \nabla\left(\bar{r} a_{p}\right), \\
c_{1 b}=\eta\left(\frac{1}{b_{\phi}} \nabla^{2} b_{\phi}-\frac{1}{\bar{r}^{2}}\right)-\frac{\bar{r}}{b_{\phi}} \boldsymbol{v}_{p} \cdot \nabla\left(\frac{b_{\phi}}{\bar{r}}\right), \\
c_{2 a}=\frac{\bar{r}}{b_{\phi}}\left[\nabla \times\left(a_{p} \hat{\boldsymbol{e}}_{\phi}\right)\right] \cdot \nabla \Omega, \\
c_{2 b}=\frac{b_{\phi}}{a_{p}} \\
c_{3 b}=\frac{\gamma b_{\phi}^{2}}{8 \pi \rho},
\end{gathered}
$$

where $a_{p}$ and $b_{\phi}$ indicate mean profiles of the spatial fields.
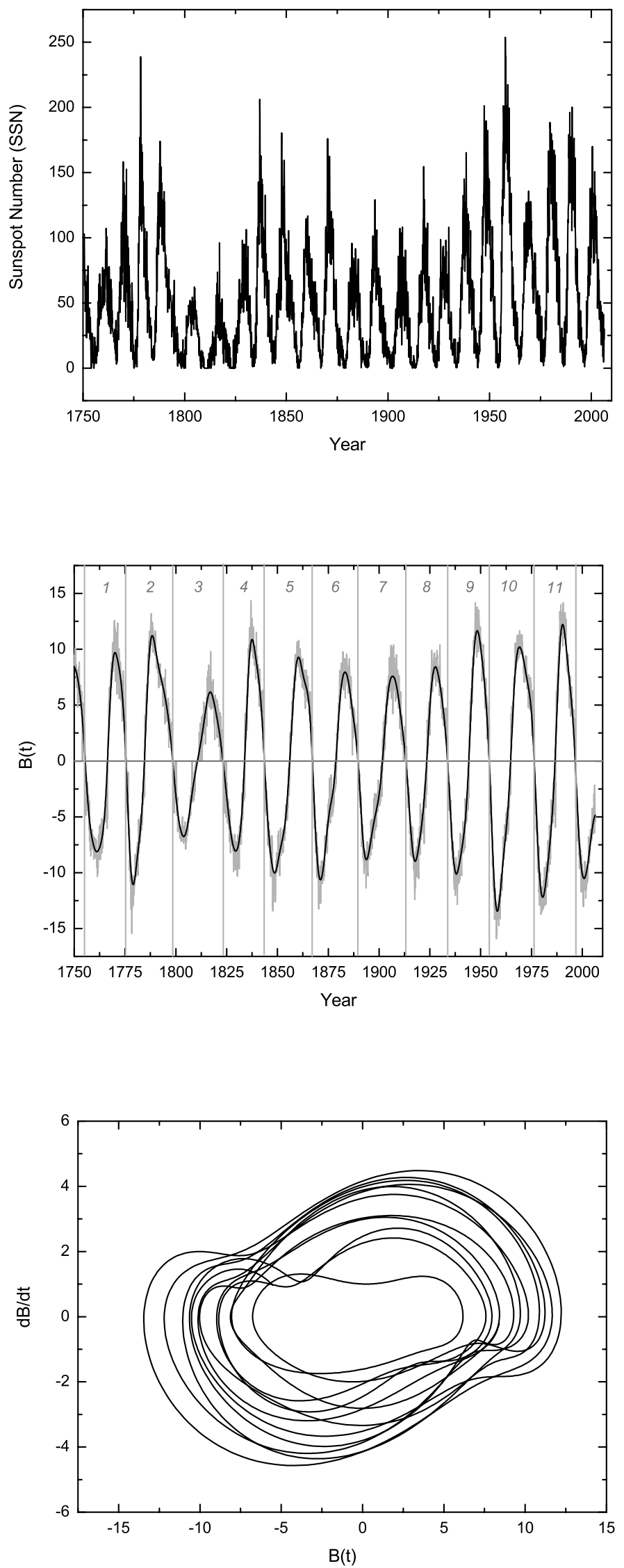

FIG. 1.-Top: Monthly sunspot number since 1750. Middle: Magnetic field $B(t)$ with the field sign inverted for each successive cycle. The black line represents the smoothed signal superposed on the original signal in gray. The individual magnetic cycles considered are also numbered. Bottom: Phase-space diagram for the smoothed $B(t)$. 
TABLE 1

Fit Parameters

\begin{tabular}{|c|c|c|c|}
\hline Cycle & $\omega$ & $\mu$ & $\xi$ \\
\hline $1 \ldots$. & $0.32558 \pm 0.00601$ & $0.16986 \pm 0.01644$ & $0.01211 \pm 0.00094$ \\
\hline ………......... & $0.30148 \pm 0.00464$ & $0.16411 \pm 0.0131$ & $0.01458 \pm 0.00086$ \\
\hline . & $0.26167 \pm 0.00415$ & $0.03511 \pm 0.02531$ & $0.0361 \pm 0.02005$ \\
\hline …........................ & $0.32241 \pm 0.00437$ & $0.19239 \pm 0.0119$ & $0.01061 \pm 0.0005$ \\
\hline$\ldots \ldots .$. & $0.27592 \pm 0.00602$ & $0.15474 \pm 0.01713$ & $0.01366 \pm 0.0011$ \\
\hline ....... & $0.29906 \pm 0.00559$ & $0.11749 \pm 0.01483$ & $0.01879 \pm 0.00181$ \\
\hline ........ & $0.27818 \pm 0.00389$ & $0.16837 \pm 0.01103$ & $0.02059 \pm 0.00097$ \\
\hline ......... & $0.30845 \pm 0.00289$ & $0.17643 \pm 0.00833$ & $0.01506 \pm 0.00051$ \\
\hline 9 & $0.31108 \pm 0.00282$ & $0.21196 \pm 0.00749$ & $0.00921 \pm 0.00025$ \\
\hline 10 & $0.29867 \pm 0.00511$ & $0.16642 \pm 0.01406$ & $0.0092 \pm 0.00057$ \\
\hline 11 & $0.31828 \pm 0.00318$ & $0.17945 \pm 0.00895$ & $0.00949 \pm 0.00034$ \\
\hline
\end{tabular}

Applying $d / d t$ to equations (5) and (6) and solving the set of equations in order to find $B_{\phi}$, we obtain

$$
\begin{aligned}
\frac{d^{2} B_{\phi}}{d t^{2}}= & -\left(c_{1 a} c_{1 b}-\alpha c_{2 a} c_{2 b}\right) B_{\phi}-\left(c_{1 a}+c_{1 b}\right) \\
& \times\left(3 \frac{c_{3 b}}{c_{1 a}+c_{1 b}} B_{\phi}^{2}-1\right) \frac{d B_{\phi}}{d t}+c_{1 a} c_{3 b} B_{\phi}^{3},
\end{aligned}
$$

or in a more compact way,

$$
\frac{d^{2} B_{\phi}}{d t^{2}}+\omega^{2} B_{\phi}+\mu\left(3 \xi B_{\phi}^{2}-1\right) \frac{d B_{\phi}}{d t}-\lambda B_{\phi}^{3}=0,
$$

where $\omega^{2}=c_{1 a} c_{1 b}-\alpha c_{2 a} c_{2 b}, \mu=c_{1 a}+c_{1 b}, \xi=c_{3 b} / \mu$, and $\lambda=c_{1 a} c_{3 b}$. Using a different method, dimensional analysis, other authors found an analogous result (Mininni et al. 2001; Pontieri et al. 2003). Equation (13) corresponds to a nonlinear oscillator that mixes both van der Pol and Duffing oscillators. According to our model we now know that the toroidal component of the solar magnetic field behaves like an oscillator. This behavior was expected and is experimentally verified (see, e.g., magnetic field data from the Wilcox Solar Observatory). This oscillator shows a characteristic phase-space behavior that depends mainly on its parameters: $\omega$, the frequency; $\mu$, the asymmetry or dumping parameter; $\xi$, the scaling parameter; and $\lambda$, the Duffing parameter.

\subsection{Further Simplifications}

By symmetry we can restrict our calculations to the northern hemisphere. We consider that, at a photospheric level, $\boldsymbol{v}_{p}$ is restricted to the surface, i.e., $\boldsymbol{v}_{p}=-v_{p} \hat{\boldsymbol{e}}_{\theta}$ (poleward directed), and $\bar{r}=R_{\odot}$. We can simplify this model even more by considering only the characteristic scale of the system. This is done through dimensional analysis, i.e., by substituting $\nabla$ with $1 / l_{0}$, where $l_{0}$ is the characteristic length of interaction, as some authors have suggested (Mininni et al. 2001; Pontieri et al. 2003). These simplifications yield

$$
\begin{aligned}
c_{1 a} & =\eta\left(\frac{1}{a_{p}} \nabla^{2} a_{p}-\frac{1}{\bar{r}^{2}}\right)-\frac{1}{\bar{r} a_{p}} \boldsymbol{v}_{p} \cdot \nabla\left(\bar{r} a_{p}\right) \\
& =\frac{\eta}{l_{0}^{2}}-\frac{\eta}{R^{2}}+\frac{v_{p}}{l_{0}}, \\
c_{1 b} & =\eta\left(\frac{1}{b_{\phi}} \nabla^{2} b_{\phi}-\frac{1}{\bar{r}^{2}}\right)-\frac{\bar{r}}{b_{\phi}} \boldsymbol{v}_{p} \cdot \nabla\left(\frac{b_{\phi}}{\bar{r}}\right) \\
& =\frac{\eta}{l_{0}^{2}}-\frac{\eta}{R^{2}}+\frac{v_{p}}{l_{0}},
\end{aligned}
$$

$$
c_{2 a}=\frac{\bar{r}}{b_{\phi}}\left[\nabla \times\left(a_{p} \hat{\boldsymbol{e}}_{\phi}\right)\right] \nabla \Omega=\frac{a_{p} R \Omega}{b_{\phi} l_{0}^{2}} .
$$

So, by applying these results to the condensed spatial factors we get

$$
\begin{gathered}
\omega^{2}=c_{1 a} c_{1 b}-\alpha c_{2 a} c_{2 b}=\left(\frac{\mu}{2}\right)^{2}-\frac{\alpha R \Omega}{l_{0}^{2}}, \\
\mu=c_{1 a}+c_{1 b}=2\left(\frac{v_{p}}{l_{0}}+\frac{\eta}{l_{0}^{2}}-\frac{\eta}{R^{2}}\right) \\
\xi=\frac{c_{3 b}}{c_{1 a}+c_{1 b}}=\frac{\gamma b_{\phi}^{2}}{4 \pi \rho \mu} \\
\lambda=c_{1 a} c_{3 b}=\frac{\mu \gamma b_{\phi}^{2}}{16 \pi \rho} .
\end{gathered}
$$

\section{PHASE-SPACE RECONSTRUCTION AND ANALYSIS METHODOLOGY}

Since in $\S 2$ we reduced the classical dynamo action to a loworder system based on $B_{\phi}$, we now try to find a way to study this magnetic field component. According to dynamo theories, the sunspot number, SSN, is proportional to the magnetic energy that erupts at the solar surface, i.e., the square of the magnetic field. Thus, we can construct a time series that exhibits a behavior analogous to the magnetic field's and is proportional to SSN by assuming $B(t) \propto \pm \sqrt{\mathrm{SSN}}$, where the sign follows the approximate 11 year inversion of the field's polarity (Bracewell 1953). Moreover, since sunspots are a direct consequence of the toroidal magnetic field that erupts at the surface, this $B(t)$ can be more correctly interpreted as proportional to the toroidal magnetic field component ( Tobias et al. 1995).

In this study, we used the monthly average international SSN from 1750 to the present. The identification of the minima and maxima for each solar cycle was the same one suggested in the database where the data came from $\left(\mathrm{NOAA}^{3}\right)$. In order to create a phase space, we also need to compute $d B / d t=[B(t+\Delta)-B(t-\Delta)] / 2 \Delta$, where in our case we chose $\Delta=6$ months for the time step. We smoothed the SSN signal (using a fast Fourier transform filter) in order to clear small-timescale sharp perturbations (smaller than 2 yr approximately), leaving only the average behavior of the cycle. In Figure 1 we show the results from this procedure.

The resulting phase-space diagram seems to indicate that the evolution of the solar cycle behaves like a low-order dynamical

\footnotetext{
${ }^{3}$ See ftp://ftp.ngdc.noaa.gov/STP/SOLAR_DATA/SUNSPOT_NUMBERS.
} 

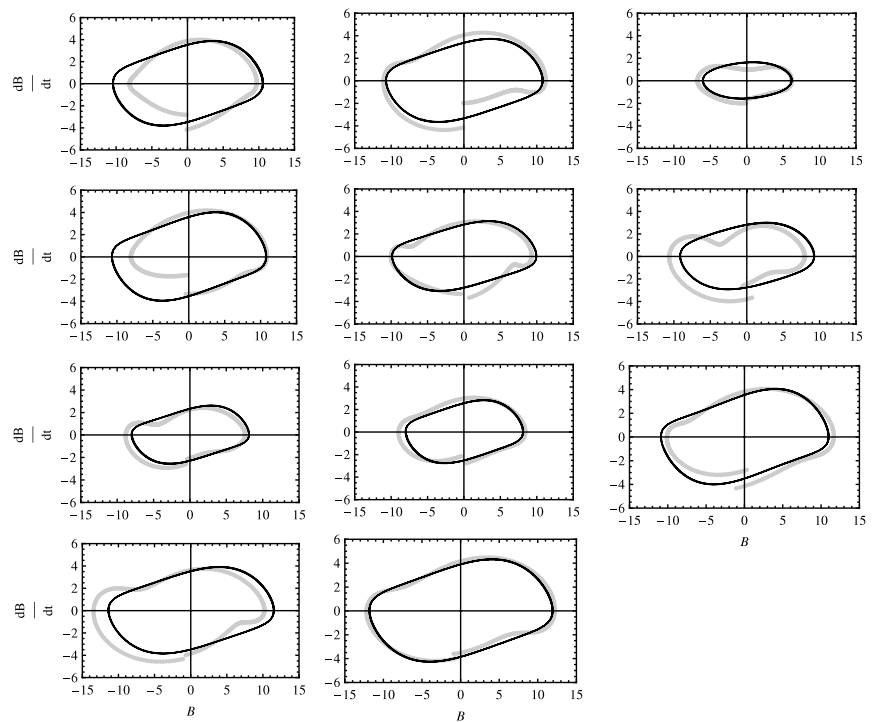

FIG. 2.- Gray dots correspond to the experimental data $B(t)$, and the black line corresponds to the fitted model. Each graph represents an individual cycle.

system. It can be interpreted as if the system is constantly readjusting to an equilibrium solution that stubbornly refuses to set still. For this case, according to our model, the solution should be an attractor, i.e., a limit closed curve in the phase space.

As mentioned in $\S 2$, this phase space represents the solutions of a nonlinear oscillator that mixes both van der Pol and Duffing oscillators. From the classical point of view of dynamical systems, the degree of randomness that the phase space (see Fig. 1, bottom) presents in itself qualifies the system as having stochastic or chaotic behavior. However, we know that, on average, the system presents a cyclic behavior, the solar cycle itself. Therefore, our approximative model should at least be able to capture some of these well-behaved solutions. Also, according to our model, we know that the characteristic parameters, $\omega, \mu$, etc., that we previously defined as spatial coefficients, are not strictly constants. They depend on the characteristics of the background (plasma velocity, diffusivity, etc.) which in turn can evolve slowly over time. Having in mind this slow evolution in time, we assume that each solar cycle corresponds to an equilibrium solution for this dynamical system, characterized by a specific set of parameters. Although the background parameters might change continuously in time, our assumption implies a discretization of the presented results.

This leads us to the following question: which background parameter is the more likely to account for the differences seen between consecutive cycles? To find an answer to this we focus our attention on the asymmetry parameter $\mu$. Besides the simple dependence it has on the background quantities (see eq. [18]), in this type of oscillator, this parameter usually indicates the strength of the nonlinear damping and, consequently, the system's energetic behavior. This is also the parameter that controls the asymmetry between the rising and falling parts of the peaks, which is also commonly seen in the solar cycle.

In order to study the slow evolution of the background parameters present in $\mu$, we divided our $B(t)$ series into individual magnetic cycles and fitted each one individually in the phase space. We used the fact that equation (13) can be described using the dynamical system's standard form, and in an analogous way to Mininni et al. (2001) and using a least-squares fit method (with the Levenberg-Marquardt algorithm), we are able to find the parameters $\omega, \mu, \xi$, and $\lambda$ that best fitted each cycle's phase

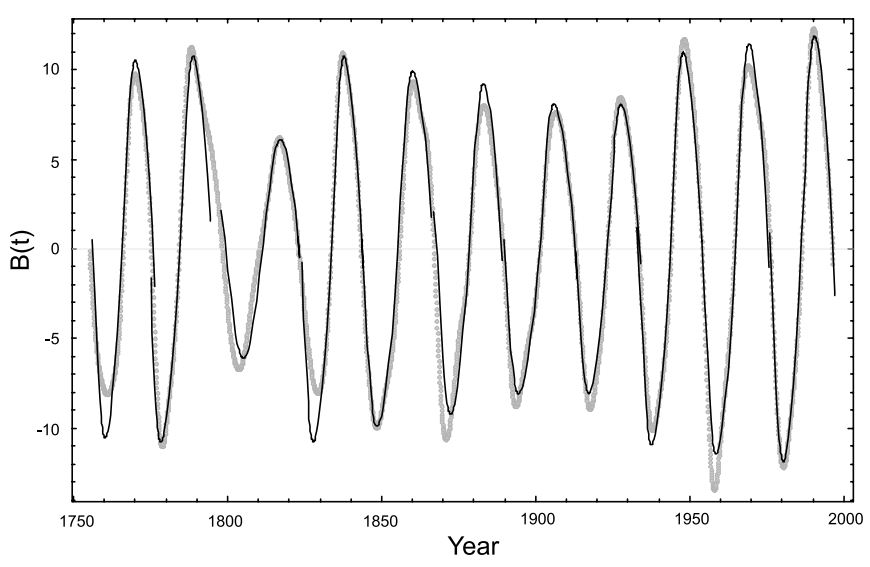

FIG. 3.-Fitted result for each cycle (lines) superposed on the smoothed $B(t)$ series (gray dots).
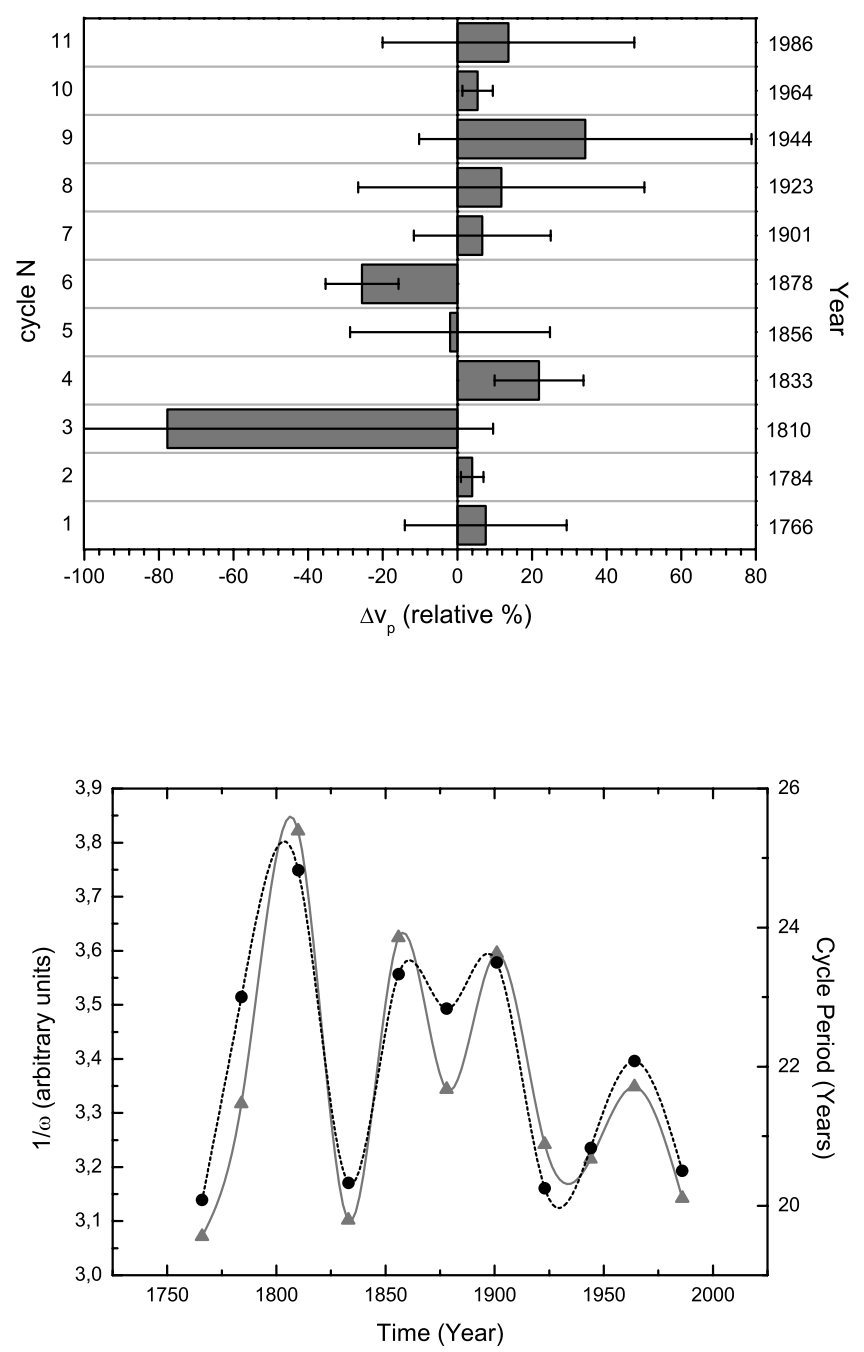

FIG. 4.-Top: Inferred variation of $v_{p}$ relative to its mean value for each considered magnetic cycle. Bottom: Black circles connected by dashed line represent the real period of each magnetic cycle. Gray triangles connected by thick line show the period $(1 / \omega)$ inferred from the fits to the data. 
space (see Table 1). The fit values for $\lambda$ are at least 2 orders of magnitude smaller than all the other parameters, and we neglect them for now. In Figures 2 and 3 we can see a comparison of the overall results of several fits in the phase space and superposed on the experimental data.

\section{MERIDIONAL CIRCULATION VARIATIONS}

An interesting fact is that, from all the quantities present in $\mu$, $v_{p}$ is, in our opinion, the more likely to change over the solar cycle timescale. Besides $v_{p}, \mu$ also depends on $l_{0}$ and $R$ (constants of the system by definition) and $\eta$, the magnetic diffusivity. The latter is usually defined as $\eta=\Lambda+\Phi$, where $\Lambda$ represents the molecular magnetic diffusivity and $\Phi$ is the turbulent magnetic diffusivity. Usually, $\Lambda$ is much smaller than $\Phi$ and in most of the cases it can be neglected. Since, to our knowledge, there is no evidence that turbulence changes its regime through the solar cycle, it is plausible to assume a constant $\eta$ in the considered time frame. Therefore, if we study the evolution of $\mu$ from cycle to cycle, we can, in a first approximation, assume that it directly reflects changes in $v_{p}$.

Figure 4 shows the deduced behavior of $v_{p}$ that is inferred from this low-order model. It represents the relative variation of the meridional circulation, $\Delta v_{p}$, at the surface. As a validation test, we also present the period from each cycle calculated using our methodology and compare it to the experimental measured values. The overall behavior is the same leading us to believe that the curve for $\Delta v_{p}$ is acceptable.

A comparison between the two graphs of Figure 4 also shows that there is an anticorrelation between $v_{p}$ and the cycle's period. This is in agreement with the evidence presented in Hathaway et al. (2003) and can be explained by the fact that a faster meridional circulation advects the poloidal field into the convection zone more efficiently decreasing the cycle's period. It is this poloidal field, which is transported to the bottom of the convection zone, that will be stretched by differential rotation and transformed into toroidal field of the next cycle. The amount of poloidal field inserted into the convection zone may have some influence on the amplitude of the following cycle (Choudhuri et al. 2007; Dikpati et al. 2006). From these arguments we can deduce that meridional circulation influences the period and amplitude of the solar cycle.

Varying meridional circulation is not a new idea, but our methodology can give us some clues about how it varied in the past. Also, evidence for a varying meridional circulation can be found in the literature (González Hernádez et al. 2006; Hathaway et al. 2003), further supporting our results.

\section{DISCUSSION AND CONCLUSIONS}

We began with the standard equations for a mean field axisymmetric $\alpha-\Omega$ dynamo and simplified it in order to obtain a low-order dynamo model. The temporal behavior of the toroidal component of the magnetic field was derived and corresponds to a modified van der Pol/Duffing oscillator. We used the sunspot number between 1750 and 2000 to construct the phase space of a quantity, $B(t)$, that behaves in an analogous ways to the toroidal component of the solar magnetic field. The resulting diagram (Fig. 1) indicates that $B(t)$ can be interpreted as a dynamical system that tends to an equilibrium solution that changes over time (this is the reason for some of the trajectories crossing in the phase space). Afterward we divided our $B(t)$ data series into individual solar magnetic cycles $(\approx 22 \mathrm{yr})$ and fitted each one of them individually to our model. This was done under the as- sumption that each solar cycle corresponds to an equilibrium trajectory on the phase space (Passos \& Lopes 2008). The values obtained through the fits give us some information about the physical parameters that are involved in the spatial coefficients. The different fit parameters obtained from cycle to cycle illustrate the evolution of the background quantities.

We chose one of the parameters, $\mu$, and studied its dependence on some background quantities, namely, magnetic diffusivity and meridional circulation. In this work we consider that the magnetic diffusivity does not change within the cycle timescale, and we further investigated the possibility that the variability observed in the phase space might be explained by a varying meridional circulation, $v_{p}$.

Concerning our methodology, we would like to point out that when the shape of the cycle in the phase space is very irregular (far form a van der Pol), the fitting process becomes unstable and the initial fitting values must be carefully introduced. In our case this happened mainly in magnetic cycles 3 and 6 . In the case of cycle 3 , the $80 \%$ deviation from average and the big error bar indicate that the fitting process probably cannot be applied here. Possibly the truncated model used is too simple to account for all existing mechanisms and simply cannot handle this extreme behavior. Although, if one believes in the values obtained with the used model for cycle 3 , this would mean that $\mu$, in that case, did not depend directly on $v_{p}$. In this particular case, the value of $\xi$ (see Table 1) is of the same order of magnitude. In the context of our model, this would mean that cycle 3 had a special dependence on the magnetic buoyancy term (see eqs. [13] and [19]). In this case, one can only try to speculate a possible explanation. This episode could be related with the intensity of the magnetic field in cycle 3 influencing the rising of the flux tubes that originate sunspots. These two cycles ( 3 and 6 ) have in common the fact that they are small when compared to their preceding ones, and interestingly, this odd behavior can be correlated to high solar activity episodes during the previous cycle. The odd behavior that begins around the end of magnetic cycle 2 (near 1788) and continues on to cycle 3 is associated with evidence of high solar activity based on auroral observations at low geomagnetic amplitudes (Vaquero \& Trigo 2006). For cycle 6, the odd behavior began at the end of cycle 5, near 1859, the year which is associated to a super solar storm that caused problems in telegraph lines all over Europe and the United States. These events occurred during the beginning of the descendant phase of the sunspot cycle which corresponds to the inflection present in the top left quadrant and bottom right quadrant of the phase space. In the scope of our simplified model, this could suggest that, when we have a very active Sun and much of the magnetic energy is released, e.g., through magnetic reconnection (associated with flares or other phenomena), the magnetic field of the following cycle has lower intensity. This low-intensity field then does not spread out up through the chromosphere and corona, confining the field more closely to the photosphere. Then the Lorentz feedback of the field acts more actively on the plasma flows and the meridional circulation slows down. Since the meridional circulation acts as a clock controlling the cycle length, this also could explain the increase in the period. This scenario is also valid for changes in the differential rotation of the Sun and agrees with previous works (Javaraiah 2003; Antia et al. 2008).

Finally, we would like to note that although the construction of the time series, $B(t)$ might be questionable, the truth is that experimental measurements from the Wilcox Solar Observatory show a good agreement between $\mathrm{SSN}$ and $B_{\phi}$. The phase-space 
analysis presented here, in addition to the more developed dynamo model, can be used in more refined ways and should be perceived as a technique capable of using existing data to study the evolution of some solar parameters.
We would like to thank the anonymous referee for the useful comments that helped improve this work and the financial support of "Fundação para a Ciência e Tecnologia" and "Fundação Calouste Gulbenkian."
Antia, H. M., Chitre, S. M., \& Gough, D. O. 2008, A\&A, 477, 657

Babcock, H. W. 1961, ApJ, 133, 572

Berhanu, M., et al. 2007, European Phys. Lett., 77, 59001

Bracewell, R. N. 1953, Nature, 133, 512

Charbonneau, P. 2005, Living Rev. Sol. Phys., 2, 2

Chatterjee, P., Nandy, D., \& Choudhuri, A. R. 2004, A\&A, 427, 1019

Choudhuri, A. R., Chatterjee, P., \& Jiang, J. 2007, Phys. Rev. Lett., 98, 131103

Dikpati, M., \& Charbonneau, P. 1999, ApJ, 518, 508

Dikpati, M., de Toma, G., \& Gilman, P. A. 2006, Geophys. Res. Lett., 33, L05102

Dubrulle, B., Blaineau, P., Mafra Lopes, O., Daviaud, F., Laval, J.-P., \& Dolganov, R. 2007, New J. Phys., 9, 308

Giles, P. M., Duval, T. L., Jr., Scherrer, P. H., \& Bogart, R. S. 1997, Nature, 390,52

González Hernádez, I., Komm, R., Hill, F., Howe, R., Corbard, T., \& Haber, D. A. 2006, ApJ, 638,576

Haigh, J. D. 1996, Science, 272, 981

\section{REFERENCES}

Harvey, K. L., \& White, O. R. 1999, ApJ, 515, 812

Hathaway, D. H., Nandy, D., Wilson, R. M., \& Reichmann, E. J. 2003, ApJ, 589,665

Javaraiah, J. 2003, Sol. Phys., 212, 23

Livingston, W. C. 1982, Nature, 297, 208

Marsh, N., \& Svensmark, H. 2003, Space Sci. Rev., 107, 317

Mininni, P. D., Gomez, D. O., \& Mindlin, G. B. 2001, Sol. Phys., 201, 203

Nandy, D., \& Choudhuri, A. R. 2002, Science, 296, 1671

Ossendrijver, M. 2003, A\&A Rev., 11, 287

Passos, D., \& Lopes, I. P. 2008, Sol. Phys., in press

Pontieri, A., Lepreti, F., Sorriso-Valvo, L., Vecchio, A., \& Carbone, V. 2003, Sol. Phys., 213, 195

Priest, E. R. 1984, Solar Magnetohydrodynamics (Dordrecht: Reidel)

Tobias, S. M., Weiss, N. O., \& Kirk, V. 1995, MNRAS, 273, 1150

Vaquero, J. M., \& Trigo, R. M. 2006, Sol. Phys., 235, 419

Wilmot-Smith, A. L., Martens, P. C. H., Nandy, D., Priest, E. R., \& Tobias, S. M. 2005, MNRAS, 363, 1167 